\title{
Study on Objective Integrated Control of New Energy Power Projects based on Reliability Theory
}

\author{
Yunna Wu, Zezhong Li*, Lirong Liu \\ School of Economics and Management, North China Electric Power University, Beijing, China \\ No. 2 Beinong road, Huilongguan,Changping district, Beijing, China, +86-18810416423 \\ *Corresponding author, e-mail: shimaotianjie001@126.com
}

\begin{abstract}
Based on the research status of objective control theory of new energy power projects, analysed the system components of power projects, proposed the subsystem reliability control theory directed at four objectives, gave reliability control standards and calculation methods of four objectives, obtained the objective integrated method of subsystem reliability, used disjoint minimal path sets method to deal with the minimal path sets in the project construction process, proposed system reliability control theory of new energy power projects, then combined the known reliability control standards to assess project reliability, finally established objective integrated control model of new energy power projects based on reliability theory. Finally an simple example proves that the proposed objective integrated control model is simple and practical.
\end{abstract}

Keywords: new energy power projects, reliability theory, project objective, integrated control

Copyright @ 2013 Universitas Ahmad Dahlan. All rights reserved.

\section{Introduction}

With the rapid development of new energy power industry, each new energy power enterprise often runs multiple power projects every year. And with the in-depth development of new energy, the number of new energy power projects is increasing rapidly, so that how to manage them effectively becomes an urgent problem. So far, in product manufacturing, power systems, structural engineering and other fields, the reliability control models have been established in progression. However, in terms of objective integrated control model of new energy power projects, domestic and foreign scholars involved less. In order to make construction results more in line with requirements, based on the reliability theory and the four major goals of quality, cost, schedule, and safety in project management, this paper studies objective integrated control problem of new energy power projects and puts forward a objective integrated method to implement project objective management.

\section{System Analysis of New Energy Power Projects}

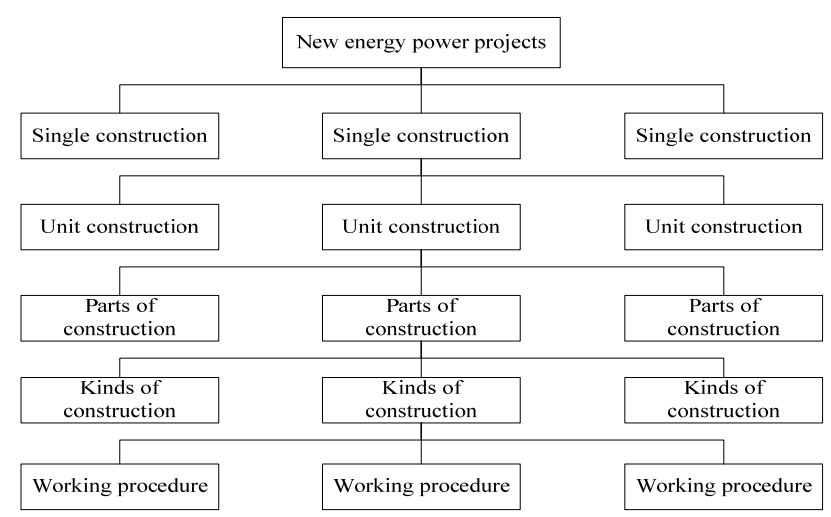

Figure.1. Construction Decomposition System of New Energy Power Projects 
Implementing project management must use the principles of system engineering to analyze the relationships between internal system and external system, total goals and subgoals, etc. A construction management process of new energy power projects can be regarded as a complex network system, and consists of single construction, unit construction, parts of construction, kinds of construction, working procedure [1], as shown in Figure 1. Then based on the related theories of network system reliability, considering the relationship between the four goals of each subsystem, we can calculate the reliability of entire network system, so that new energy power enterprises can control projects' objectives and implement project management better and better according to the calculated results.

\section{Reliability Calculation of Parts of Construction based on Four Major Objectives}

We often attribute engineering construction project objectives to three main objectives of quality, cost and schedule. However, safety problem is the premise of the engineering construction, and project safety in the building process will have direct impact on the reliability of the entire project [2]. The four main objectives can reflect the project management level comprehensively. Therefore, based on the four objectives, this paper conducts objective reliability control on new energy power projects. This paper selects parts of construction as the minimum calculation unit, and gets the disjoint minimal path sets of the entire network system. According to the reliability of subsystem-parts of construction, we can calculate the reliability of new energy power projects.

Considering the four objectives of quality, schedule, cost and safety, the basic ideas of determining the reliability of parts of construction are as follows:

First of all, consider different objectives characteristics, take different approaches to calculate each objective reliability of parts of construction.

Secondly, use systems engineering ideas to deal with this kind of problems and regard the four major objectives as a series system [3], as shown in Figure 2.

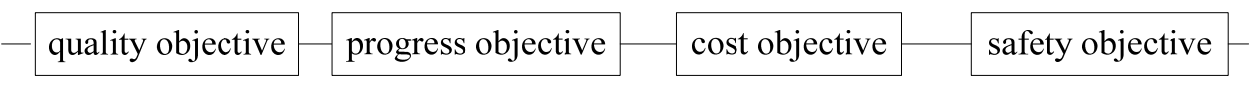

Figure 2. Series Structure Diagram of Four Goals reliability [4]:

According to the reliability formula (1) of series system, we can get the subsystem

$$
R\left(x_{i}\right)=R(Q) \times R(C) \times R(T) \times R(S)
$$

Among, $R\left(x_{i}\right)$ represents the reliability of parts of construction, $R(Q), R(C), R(T)$, and $R(S)$ represent the reliability of the four goals of quality, cost, schedule and safety respectively.

\subsection{Quality Reliability $R(Q)$}

There are many different kinds of factors affecting project quality, but from the point of view of quality management, there are five main areas, including human factor, material, machine, method, and environment, and these five areas are referred to as 4M1E [5]. And the five factors include multiple evaluation factors, as shown in Table 1.

After determining their respective evaluation factors of five influencing factors, experts give their scores and conduct fuzzy comprehensive evaluation. In evaluation on quality factors, there are only two grades of qualified and unqualified. We select $L$ to stand for quality grade, and aimed at evaluation factor $j$ of influencing factor $i$, when the unqualified proportion of experts scores is more than $1 / 3, L$ equals to 0 ; or $L$ equals to 1 [6]. 
Table 1. Influencing Factors of Project Quality

\begin{tabular}{|c|c|c|}
\hline & Influencing factors & Evaluation factors \\
\hline & \multirow{4}{*}{ Human factor $A_{1}$} & Cultural level $\mathrm{a}_{11}$ \\
\hline & & Decision-making capacity $\mathrm{a}_{13}$ \\
\hline & & Management ability $\mathrm{a}_{14}$ \\
\hline & & Professional ethics $\mathrm{a}_{16}$ \\
\hline & \multirow{2}{*}{ Material $\mathrm{A}_{2}$} & Material selection $\mathrm{a}_{21}$ \\
\hline & & Material quality $a_{22}$ \\
\hline \multirow{7}{*}{$\begin{array}{c}\text { Project quality } \\
\text { objective }\end{array}$} & & Process equipment and various tools composing of engineering entity \\
\hline & Machine $A_{3}$ & $\begin{array}{l}a_{31} \\
\text { Various types of machinery and equipment used in construction } \\
\text { process } a_{32}\end{array}$ \\
\hline & \multirow{4}{*}{ Method $\mathrm{A}_{4}$} & Planning method $a_{41}$ \\
\hline & & Controlling method $\mathrm{a}_{42}$ \\
\hline & & Organizational method $\mathrm{a}_{43}$ \\
\hline & & $\begin{array}{l}\text { Leadership method } \mathrm{a}_{44} \\
\text { Process method } \mathrm{a}_{45} \\
\text { Operation method } \mathrm{a}_{46} \\
\text { Construction method } \mathrm{a}_{47}\end{array}$ \\
\hline & Environment $A_{5}$ & Project management conditions $\mathrm{a}_{54}$ \\
\hline
\end{tabular}

Since the weights of influencing factors are not equal, we firstly give their weights before we determine the reliabilities of the quality objectives. $A=[0.30,0.18,0.17,0.18,0.17]$. Establish the reliability calculation model for quality objective:

$$
R(Q)=\sum A_{i} C_{i}
$$

Among formula (2), $A_{i}$ represents the weights of five impact factors, $C_{i}$ represents the proportion of qualified factors and all the factors in $i$-th influencing factor, $i=1,2, \cdots, 5$

\subsection{Schedule Reliability $R(T)$}

Generally speaking, we often use earned value method to calculate the reliability of schedule and cost objective. By analyzing the differences between the implementation situation of project objectives and the implementation situation of project expectations, we can evaluate project management performance. The schedule reliability calculation model of parts of construction is shown in Equation (3):

$$
R(T)=1-\frac{\mid \text { Actual completion time-Scheduled completion time } \mid}{\text { Scheduled completion time }}
$$




\subsection{Cost reliability $R(C)$}

The reliability calculation method of cost objective is similar to that of schedule objective. The cost reliability calculation model of parts of construction is shown in Equation (4):

$$
R(C)=1-\frac{\mid \text { Actual cost-Planned cost } \mid}{\text { Planned cost }}
$$

\subsection{Safety Reliability $R(S)$}

In the reliability assessment of construction projects in the past, they are not related to safey control, however, safey control is the only important goal related to personal safety. According to the seriousness of possible consequences, divide safety level, and give the reliability range of safety objective [7]. Based on Table 2, this paper uses interpolation method to determine the reliability value of safety objective.

Table 2. Safety Level Division Standard and Reliability Assignment

\begin{tabular}{ccc}
\hline Safety level & Standard of division & Reliability assign \\
\hline Grade 1 & no injuries and deaths & $0.80-1$ \\
Grade 2 & $\begin{array}{c}\text { the economy loss beyond } 0.4 \% \text { of the total cost } \\
\text { The injuries and deaths rate beyond } 1 \%\end{array}$ \\
the economy loss beyond $2 \%$ of the total cost & $0.50-0.79$ \\
Grade 3 & $\begin{array}{c}\text { The injuries and deaths rate over 1\% or have deaths the economy loss } \\
\text { over 2\% of the total cost }\end{array}$ & $<0.50$ \\
\hline
\end{tabular}

\subsection{Reliability of Parts of Construction $R(P)$}

Based on the above reliability calculation results of four objectives, the reliability calculation model of parts of construction is shown in Equation (5):

$$
R(P)=R(Q) \times R(T) \times R(C) \times R(S)
$$

\section{System Reliability Calculation and Allocation}

\subsection{Minimal Path Sets of New Energy Power Projects}

There are several different calculation methods of minimal path sets, and the relatively mature methods currently include: contact matrix method, determinant method, node search method. Combined with the characteristics of new energy power projects and the difficulty degree of various calculation methods, the paper selects the determinant method to calculate the minimal path sets.

Assuming the given system network matrix $\mathrm{C}$, the concrete steps of determinant method are as follows:

1) Construct a unit matrix $U$ which has the same dimension with the network matrix $C$, add $\mathrm{U}$ to the matrix $\mathrm{C}$, get Matrix $\mathrm{Z}, \mathrm{Z}=\mathrm{U}+\mathrm{C}$;

2) Delete the rows corresponding to the input and output points in matrix $Z$, thus get a new matrix $\mathrm{W}$;

3) According to determinant calculation principles, expand the matrix $W$ to algebra form, and all the values take positive, thus get the minimal path sets of network system S.

\subsection{Disjoint Minimal Path Sets Calculation Regulations}

(1) Assume that the system has $n$ minimal path sets, they are $K_{1}, K_{2}, \ldots, K_{n}$, and they may intersect. For the whole minimal path sets $S=K_{1}+K_{2}+\ldots+K_{n}$, the disjoint process has the following formulas:

$$
S=K_{1}+K_{2}+\ldots+K_{n}=K_{1}+\overline{K_{1}} K_{2}+\overline{K_{1}} \overline{K_{2}} K_{3}+\ldots+\overline{K_{1}} \overline{K_{2}} \ldots \overline{K_{n-1}} K_{n}
$$

(2) For a parallel structure $S$ composed of $\mathrm{n}$ units, expression is as follows: 


$$
S=x_{1}+\overline{x_{1}} x_{2}+\ldots+\prod_{i=1}^{n-1} \overline{x_{i}} \cdot x_{2}
$$

$X_{i}$ represents the normal working probability of I'th arc (unit), namely its reliability; $\overline{x_{i}}=1-x_{i}$ represents the abnormal probability, namely its unreliability.

(3) Morgan theorem disjoint expressions:

$$
\left\{\begin{array}{l}
\overline{x_{1} x_{2} \ldots x_{n}}= \\
\overline{x_{1}}+x_{1} \overline{x_{2}}+\prod_{i=1}^{n-1} x_{i} \cdot \overline{x_{n}} \\
\overline{x_{1}+\overline{x_{1}} x_{2} \ldots \prod_{i=1}^{n-1} \overline{x_{i}} \cdot x_{n}} \\
=\overline{x_{1}} \cdot \overline{x_{2}} \ldots \overline{x_{n}}
\end{array}\right.
$$

(4) General rules of simplifying process:

$$
\begin{aligned}
& \text { Commutative }\left\{\begin{array} { l } 
{ A + B = B + A } \\
{ A B = B A }
\end{array} \quad \text { Absorption law } \left\{\begin{array}{l}
A+A B=A \\
A(A+B)=A
\end{array}\right.\right. \\
& \text { Associative law }\left\{\begin{array}{l}
A+(B+C)=(A+B)+C \\
A(B C)=(A B) C
\end{array}\right. \\
& \text { Distributive law }\left\{\begin{array}{l}
A(B+C)=A B+A C \\
A+B C=(A+B)(A+C)
\end{array}\right. \\
& \text { Morgan law }\left\{\begin{array} { l } 
{ \overline { A + B } = \overline { A } \cdot \overline { B } } \\
{ \overline { A B } = \overline { A } + \overline { B } }
\end{array} \quad \text { Equal law } \left\{\begin{array}{l}
A+A=A \\
A \cdot A=A
\end{array}\right.\right.
\end{aligned}
$$

Coverage law $A+B=A+\bar{A} B$

\subsection{System Reliability Calculation}

After disjoint treatment on minimal path sets, we get all disjoint minimal path sets $L_{1}, L_{2}, \ldots, L_{m}$, at least one smooth path set, the system will be able to work properly, that is, the system is normal $S=\bigcup_{i=1}^{m} L_{i}$. System reliability is as follows:

$$
R_{S}=P(S)=P\left(\sum_{i=1}^{m} L_{i}\right)
$$

\subsection{Reliability Allocation}

For an established objective integrated control system model of new energy power projects, if the calculated system reliability cannot meet the specified reliability objective value, we should allocate reliability value again. Starting from the entire system, allocate the reliability target value to various subsystems, and each subsystem will have a new reliability allocation index, and in accordance with this reliability index we can have a real-time control on project objectives. Once actual reliability calculation results achieve the assigned index requirements, it will be able to achieve the reliability goals of the whole system, and this can also show the objective integrated control model has some practical significance [8]. The usual reliability allocation methods include: equal-distribution method, redistribution method, and system failure rate expected value method, AGREE method, and minimum cost allocation method, etc. This paper selects the redistribution method to conduct the reliability redistribution. The basic ideas of reliability redistribution: improve the reliability of units whose original reliability is low to a 
certain value, while the originally higher unit reliability remains unchanged. The concrete steps are as follows:

1. Arrange the reliability values of respective units in ascending order.

$$
R_{1}<R_{2}<\cdots<R_{m}<R_{m+1}<\cdots<R_{n}
$$

2. Improve the reliability values of $R_{1}, R_{2}, \cdots, R_{m}$ who have the lower reliability to a certain value $R_{0}$, while the original higher reliability values of $R_{m+1}, \cdots, R_{n}$ remain unchanged, then the system reliability value is as follows:

$$
R^{*}=R_{0}^{m} \prod_{i=m+1}^{n} R_{i}
$$

3. Determine $m$ and $R_{0}$, that is, determine the units whose reliability need to be improved and improve the reliability to what extent. The value of $R_{0}$ can be obtained by the formula (13).

$$
R_{0}=\left(\frac{R^{*}}{\prod_{i=m+1}^{n} R_{i}}\right)^{\frac{1}{m}}<R_{m+1}
$$

The value of $m$ can be obtained by the inequality formula (14).

$$
R_{m}<R_{0}=\left(\frac{R^{*}}{\prod_{i=m+1}^{n} R_{i}}\right)^{\frac{1}{m}}<R_{m+1}
$$

\section{Case Study}

In the construction process of a new energy power enterprise project, a unit project has 5 parts of construction, including subgrade engineering, hole engineering, pavement engineering, hole body excavation, and hole body lining, and the reliability control standard of unit project is six times of standard deviation range, that is, its reliability control standard is 0.67 , and dual-code network plan is shown in Figure 3. Then experts gives their respective scores for each parts of construction, and quality assessment results of sub-grade engineering are as follows: human factors qualified; material: material selection $a_{21}$ unreasonable; machine factors qualified; method: leadership method $a_{44}$ unqualified; environment: engineering and technical conditions $a_{53}$ unqualified. After the budget and final accounting, cost assessment results are as follows: the planned cost is 550 million yuan and the actual cost is 600 million yuan. After measurement and statistics, the schedule assessment results are as follows: the planned completion time is 300 days and the actual completion time is 320 days. The safety examination results are as follows: a fire happened and it caused economic losses of 1.28 million yuan; no injuries and deaths.

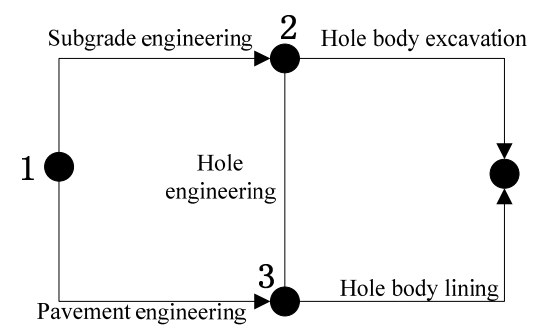

Figure 3. Double Arrow Network of a Unit Engineering of New Energy Power Projects 
Use arcs to represent each parts of construction in Figure 3 , and $x_{i}(i=1,2, \ldots, 13)$ represent the names of parts of construction, so that Figure 3 is transformed into a complex network system diagram of Figure 4.

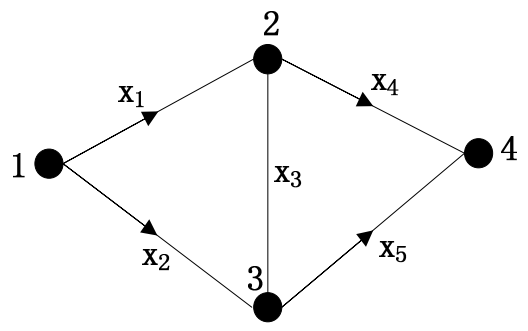

Figure 4. The Bridge Network Block Diagram of a Unit Engineering of New Energy Power Projects

\section{(1) Calculate the Reliability of the Five Parts of Construction}

According to the provided data, firstly calculate the objective reliability of sub-grade engineering:

According to the formula (2), calculate the quality reliability of sub-grade engineering:

$$
R(Q)=\sum A_{i} C_{i j}=0.30 \times 1+0.18 \times \frac{3}{4}+0.17 \times 1+0.18 \times \frac{6}{7}+0.17 \times \frac{3}{4}=0.887
$$

According to the formula (3), calculate the schedule reliability of sub-grade engineering:

$$
R(T)=1-\frac{\mid \text { Actual completion time-Scheduled completion time } \mid}{\text { Scheduled completion time }}=1-\frac{|320-300|}{300}=0.933
$$

According to the formula (4), calculate the cost reliability of sub-grade engineering:

$$
R(C)=1-\frac{\mid \text { Actual cost-Planned cost } \mid}{\text { Planned cost }}=1-\frac{|600-550|}{550}=0.909
$$

Based on safety level classification in Table 1, we can see that the safety level the subgrade engineering is in Grade 1. After the reasonable 0.618 interpolation operation, the safety reliability of sub-grade engineering is 0.924 .

According to the formula (5), calculate the reliability of sub-grade engineering:

$$
R(\text { sub - grade engineering })=R(Q) \times R(T) \times R(C) \times R(S)=0.695
$$

Similarly, we can calculate the reliability of other subsystems (parts of construction), and the results are shown in Table 3.

Table 3. Reliability of Each Subsystem (Parts of Construction)

\begin{tabular}{cccccc} 
Subsystem $X_{i}$ & $X_{1}$ & $X_{2}$ & $X_{3}$ & $X_{4}$ & $X_{5}$ \\
\hline Reliability $R_{i}$ & 0.695 & 0.875 & 0.700 & 0.650 & 0.818
\end{tabular}

(2) Use determinant method to seek minimal path sets of the network system Firstly, according to Figure 4, the network matrix is shown as follows: 


$$
C=\left[\begin{array}{cccc}
0 & x_{1} & x_{2} & 0 \\
0 & 0 & x_{3} & x_{4} \\
0 & x_{3} & 0 & x_{5} \\
0 & 0 & 0 & 0
\end{array}\right]
$$

And, $U=\left[\begin{array}{cccc}1 & 0 & 0 & 0 \\ 0 & 1 & 0 & 0 \\ 0 & 0 & 1 & 0 \\ 0 & 0 & 0 & 1\end{array}\right]$

So,

$$
Z=U+C=\left[\begin{array}{cccc}
1 & x_{1} & x_{2} & 0 \\
0 & 1 & x_{3} & x_{4} \\
0 & x_{3} & 1 & x_{5} \\
0 & 0 & 0 & 1
\end{array}\right]
$$

Delete the first column and the fourth row and get the determinant $W$ :

$$
W=\left|\begin{array}{ccc}
x_{1} & x_{2} & 0 \\
1 & x_{3} & x_{4} \\
x_{3} & 1 & x_{5}
\end{array}\right|=x_{1} x_{3} x_{5}+x_{2} x_{3} x_{4}-x_{1} x_{4}-x_{2} x_{5}
$$

So, system minimal path sets $S=x_{1} x_{3} x_{5}+x_{2} x_{3} x_{4}+x_{1} x_{4}+x_{2} x_{5}$, Or it can be expressed as $S=\left\{x_{1} x_{3} x_{5}, x_{2} x_{3} x_{4}, x_{1} x_{4}, x_{2} x_{5}\right\}$.

\section{(3) Disjoint treatment on minimal path sets}

Based on the above disjoint treatment rules of minimal path sets, we conduct disjoint treatment on minimal path sets of the building construction process, as follows:

$$
\begin{aligned}
& S=x_{1} x_{3} x_{5}+\overline{x_{1} x_{3} x_{5}} \cdot x_{2} x_{3} x_{4}+\overline{x_{1} x_{3} x_{5}} \cdot \overline{x_{2} x_{3} x_{4}} \cdot x_{1} x_{4}+\overline{x_{1} x_{3} x_{5}} \cdot \overline{x_{2} x_{3} x_{4}} \cdot \overline{x_{1} x_{4}} \cdot x_{2} x_{5} \\
& =x_{1} x_{3} x_{5}+\left(\overline{x_{1}}+x_{1} \overline{x_{3}}+x_{1} x_{3} \overline{x_{5}}\right) x_{2} x_{3} x_{4}+\left(\overline{x_{1}}+x_{1} \overline{x_{3}}+x_{1} x_{3} \overline{x_{5}}\right)\left(\overline{x_{2}}+x_{2} \overline{x_{3}}+x_{2} x_{3} \overline{x_{4}}\right) x_{1} x_{4} \\
& +\left(\overline{x_{1}}+x_{1} \overline{x_{3}}+x_{1} x_{3} \overline{x_{5}}\right)\left(\overline{x_{2}}+x_{2} \overline{x_{3}}+x_{2} x_{3} \overline{x_{4}}\right)\left(\overline{x_{1}}+x_{1} \overline{x_{4}}\right) x_{2} x_{5} \\
& =x_{1} x_{3} x_{5}+\overline{x_{1}} x_{2} x_{3} x_{4}+x_{1} x_{2} x_{3} x_{4} \overline{x_{5}}+x_{1} \overline{x_{2}} \overline{x_{3}} x_{4}+x_{1} x_{2} \overline{x_{3}} x_{4}+x_{1} \overline{x_{2}} x_{3} x_{4} \overline{x_{5}}+ \\
& \overline{x_{1}} x_{2} \overline{x_{3}} x_{5}+\overline{x_{1}} x_{2} x_{3} \overline{x_{4}} x_{5}+x_{1} x_{2} \overline{x_{3}} \overline{x_{4}} x_{5}
\end{aligned}
$$

\section{(4) Network system reliability calculation}

Apply the reliability results of each parts of construction, we can get the system reliability of unit construction, and the concrete steps are as follows:

$$
\begin{aligned}
& R_{s}=R_{1} R_{3} R_{5}+\left(1-R_{1}\right) R_{2} R_{3} R_{4}+R_{1} R_{2} R_{3} R_{4}\left(1-R_{5}\right)+R_{1}\left(1-R_{2}\right)\left(1-R_{3}\right) R_{4}+ \\
& R_{1} R_{2}\left(1-R_{3}\right) R_{4}+R_{1}\left(1-R_{2}\right) R_{3} R_{4}\left(1-R_{5}\right)+\left(1-R_{1}\right) R_{2}\left(1-R_{3}\right) R_{5}+ \\
& \left(1-R_{1}\right) R_{2} R_{3}\left(1-R_{4}\right) R_{5}+R_{1} R_{2}\left(1-R_{3}\right)\left(1-R_{4}\right) R_{5} \\
& =R_{2} R_{5}+R_{1} R_{4}+R_{1} R_{3} R_{5}+R_{2} R_{3} R_{4}-R_{1} R_{2} R_{3} R_{4}- \\
& R_{1} R_{3} R_{4} R_{5}-R_{2} R_{3} R_{4} R_{5}-R_{1} R_{2} R_{3} R_{5}-R_{1} R_{2} R_{4} R_{5}+2 R_{1} R_{2} R_{3} R_{4} R_{5} \\
& \approx 0.8837
\end{aligned}
$$


As can be seen from the above results, the reliability value of the above unit construction is 0.884 , so it is greater than the prescribed reliability standard value of 0.67 , so the unit construction of new energy power project is in the qualified status of the four-goal control, and there is no need to reallocate the reliability of each parts of construction [9]. If we have some other conditions, we can get the reliability situation of single construction, or if it is necessary, we can calculate the system reliability value of the entire new energy power project. According to the calculation results, we can conduct the objective integrated control and have better implementation of the new energy power project management.

\section{Conclusion}

Considering the four goals of quality, cost, schedule and safety comprehensively, this paper establishes the objective integred control model of ew energy power projects. The method is simple and practical, and not only it applies to parts of construction, unit construction, but also it applies to working procedure, single construction, as well as a whole new energy power projects. The model is mainly used for objective integrated control in the construction process of construction project, and can be used to evaluate the results of project construction. This method can provide guidance on project objectiveintegrated control and has a certain practical significance.

\section{Acknowledgements}

This work was financially supported by the National Natural Science Foundation of China -Non-profit Public Investment Projects coordinated supervision in the project group environment (71271085) and Philosophy and Social Sciences, Beijing Twelfth Five Year Plan Project (12JGB044), and the Common Construction Project of Beijing Central University.

\section{References}

[1] Sun Kui-zhou, Feng Hu-tian, Zhou Jin-yu. Reliability optimum design of spring in the tools storage. Telkomnika. 2012; 10(5): 1123-1129.

[2] Ning Lu, Yanping Feng, Fang Wang, etc. Research on unit project reliability control based on four targets. Journal of Xi'an University of Architecture \& Technology (Natural Science Edition). 2007; 39(5): 652-656.

[3] Ning Lu, Yufang Shi, Xuanqiang Gao, et al. Research on calculation method of sub-system reliability of construction working procedure. Journal of Xi'an University of Architecture \& Technology (Natural Science Edition). 2006; 38(3): 311-320.

[4] Liqiong Yang, Shirong Li, Bo Xu. Reliability Evaluation of Construction System for Large Projects. Journal of Chongqing University (Social Science Edition). 2012; 18(5): 64-69.

[5] Yunna Wu, Wenjun Chen. Engineering project management. Publishing house of electronics industry 2009.

[6] Li Jiejia, Liu, Daiyan, Qu Rui. The strategy of construction equipment energy-saving control. Telkomnika. 2012; 10(4): 706-712.

[7] China Association of Engineering Consultants. Quality control of construction projects. China architecture \& building press. 2003: 123-124.

[8] Weiya Jin, Kangda Zhang. Reliability Engineering. Chemical industry press 2005: 44-51.

[9] Zein Hermagasantos, Sabri Yusra, Mashar Ali. Implementation of electricity competition framework with economic dispatch direct method. Telkomnika. 2012; 10(4): 625-632. 